\title{
SWIR InGaAs/GaAsSb type-II quantum well photodetectors and spectrometers integrated on SOI
}

\author{
Ruijun Wang ${ }^{\mathrm{a}, \mathrm{b},{ }^{*}}$, Muhammad Muneeb ${ }^{\mathrm{a}, \mathrm{b}}$, Stephan Sprengel ${ }^{\mathrm{c}}$, Gerhard Boehm ${ }^{\mathrm{c}}$, Roel Baets ${ }^{\mathrm{a}, \mathrm{b}}$, \\ Markus-Christian Amann ${ }^{\mathrm{c}}$, Gunther Roelkens ${ }^{\mathrm{a}, \mathrm{b}}$
}

\author{
a. Photonics Research Group, Ghent University-imec, Sint-Pietersnieuwstraat 41, B-9000 Ghent, \\ Belgium; \\ b. Center for Nano- and Biophotonics (NB-Photonics), Ghent University, Ghent, Belgium; \\ c. Walter Schottky Institut, Technische Universität München, Am Coulombwall 4, 85748 Garching, \\ Germany.
}

\begin{abstract}
The short-wave infrared wavelength range $(2-3 \mu \mathrm{m})$ is attractive for applications in gas sensing and next-generation communication systems. Photodetectors and wavelength (de)multiplexers are key components that have to be developed for these systems. In this contribution, we report the integration of InGaAs/GaAsSb type-II quantum well photodetectors and spectrometers on the silicon photonics platform. In this photodetector epitaxial layer stack, the absorbing active region consists of 6 periods of $\mathrm{W}$-shaped quantum wells, which can also be used to realize lasers. The efficient coupling between silicon waveguides and quantum well photodetectors is realized by tapered III-V waveguides. The photodetectors have a very low dark current of $12 \mathrm{nA}$ at $-0.5 \mathrm{~V}$ bias at room temperature. The devices show a responsivity of $1.2 \mathrm{~A} / \mathrm{W}$ at 2.32 $\mu \mathrm{m}$ wavelength, and higher than $0.5 \mathrm{~A} / \mathrm{W}$ over the $2.2-2.4 \mu \mathrm{m}$ wavelength range. On the silicon-on-insulator platform we also demonstrate high performance short-wave infrared spectrometers. 8-channel spectrometers in the 2.3-2.4 $\mu \mathrm{m}$ range with a resolution of $5 \mathrm{~nm}$ and $1.4 \mathrm{~nm}$ are demonstrated, showing a cross-talk below $-25 \mathrm{~dB}$ and an insertion loss lower than $3 \mathrm{~dB}$.
\end{abstract}

Keywords: silicon photonics, short-wave infrared, photodetectors, arrayed waveguide gratings, heterogeneous integration ${ }^{1}$

\section{INTRODUCTION}

The 2-3 $\mu \mathrm{m}$ short-wave infrared (SWIR) wavelength range is of great interest for spectroscopic sensing as a lot of important industrial gases have strong absorption signatures in this wavelength range $^{1}$. For example, the wavelength range around $2.3 \mu \mathrm{m}$ offers the first water absorption free spectral window for $\mathrm{CO}$ detection. Besides, this wavelength range has also shown potential for next generation communication systems because of recently improved transmission and amplification capabilities of hollow-core photonic-bandgap fibres ${ }^{2}$. In recent years, silicon photonics has been attracting a lot of interest for the SWIR wavelength range since it can take advantage of standard complementary metal-oxide-semiconductor (CMOS) process technology to fabricate integrated photonic devices ${ }^{3,4}$. Among all of the components in integrated photonics system, arrayed waveguide gratings (AWGs) are widely used in optical communication and spectroscopy ${ }^{5}$. AWGs implemented in silicon photonicsare much more compact than the devices based on low-index-contrast material systems. AWG spectrometers operating around $2.2 \mu \mathrm{m}$ were demonstrated based on a silicon-on-insulator (SOI) strip waveguide structure ${ }^{6}$. The low resolution $(4.8 \mathrm{~nm})$ and high resolution $(1.6 \mathrm{~nm})$ AWG spectrometers have a crosstalk of $12 \mathrm{~dB}$ and $-16 \mathrm{~dB}$, and insertion loss of $2.1 \mathrm{~dB}$ and $4 \mathrm{~dB}$, respectively. Comparing with an SOI AWG operating at telecommunication wavelengths, the performance of the $2 \mu \mathrm{m}$ wavelength range SOI AWGs should be further improved ${ }^{7}$.

*Ruijun.Wang@intec.ugent.be 
In this paper, we demonstrate low insertion loss and low crosstalk AWGs based on a shallow-etched SOI waveguide platform. The low resolution $(5 \mathrm{~nm})$ and high resolution $(1.4 \mathrm{~nm})$ AWG spectrometers have a crosstalk of $-30 \mathrm{~dB}$ and -25 $\mathrm{dB}$, and insertion loss of $2.5 \mathrm{~dB}$ and $2.8 \mathrm{~dB}$, respectively.

Photodetectors are also one of the key components that should be developed for silicon photonics systems in the 2-3 $\mu \mathrm{m}$ SWIR wavelength range. Epitaxial growth of strain-relaxed GeSn on silicon can be used to achieve cut-off wavelengths beyond $2 \mu \mathrm{m}^{8}$. However, this material system has not yet been demonstrated to be integrated with silicon waveguide circuits. Recently, monolithic silicon photodetectors with responsivity of $0.3 \mathrm{~A} / \mathrm{W}$ in the $2 \mu \mathrm{m}$ wavelength range were demonstrated based on defect engineered silicon ${ }^{9}$. Integration of III-V material on silicon photonics platform by adhesive bonding or molecular bonding is an effective technology to integrate active opto-electronic devices on the silicon platform ${ }^{10}$. There are many demonstrations of heterogeneously integrated InP-based lasers and photodetectors on silicon waveguide circuits for the $1.3 \mu \mathrm{m} / 1.55 \mu \mathrm{m}$ optical communication wavelength range, however, there are very few demonstrations beyond $2 \mu \mathrm{m}$ wavelength. For the 2-3 $\mu \mathrm{m}$ wavelength range, GaSb-based type-I heterostructures can be used to make active devices. However, the heterogeneous integration process and device processing yield of GaSb-based devices lags behind that of InP. Alternatively, the emission and detection cut-off wavelength of InP-based type-II heterostructures can cover the 2-3 $\mu \mathrm{m}$ wavelength range ${ }^{11}$. Heterogeneous integration of InP-based type-II devices can take advantage of the mature InP heterogeneous integration processes. In this paper, we report the integration of InP-based type-II InGaAs/GaAsSb quantum well photodetectors on SOI. At room temperature, the heterogeneously integrated photodetectors show a dark current of $12 \mathrm{nA}$ at a reverse voltage of $0.5 \mathrm{~V}$, and a responsivity of $1.2 \mathrm{~A} / \mathrm{W}$ at $2.32 \mu \mathrm{m}$ wavelength.

\section{SWIR AWG SPECTROMETER}

The fabrication of passive SOI waveguide circuits are carried out on $200 \mathrm{~mm}$ silicon wafers in imec's CMOS pilot line. The SOI wafer consists of a $400 \mathrm{~nm}$ thick silicon device layer on a $2 \mu \mathrm{m}$ buried oxide layer. $850 \mathrm{~nm}$ wide waveguides are designed for the propagation of $2.35 \mu \mathrm{m}$ wavelength light. The silicon waveguide pattern is defined by $193 \mathrm{~nm}$ deepultraviolet (DUV) lithography. Then inductively-coupled plasma (ICP) dry etching is used to etch $180 \mathrm{~nm}$ deep silicon rib waveguide with smooth sidewalls and a vertical profile. Afterwards, a layer of $\mathrm{SiO}_{2}$ is deposited on the SOI wafer and planarized by a chemical mechanical polishing (CMP) process.

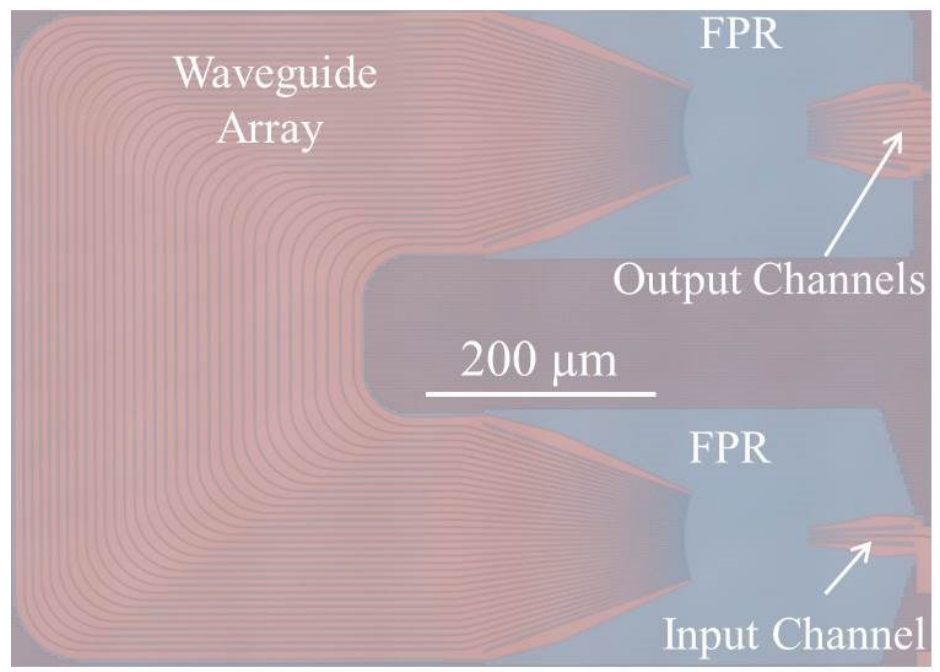

Figure 1. Microscope image of the low resolution AWG. 
Detailed information about the principle of AWG design can be found in [5]. A low resolution AWG with channel spacing of $5 \mathrm{~nm}$ and a high resolution AWG with channel spacing of $1.4 \mathrm{~nm}$ are designed for the 2.3-2.4 $\mu \mathrm{m}$ wavelength range in this work. Both AWGs have 8 channels for using. For these AWGs, we choose the following parameters for design: the number of arrayed waveguides $\mathrm{N}_{\mathrm{wg}}=32$, the length of the free propagation region (FPR) $\mathrm{L}_{\mathrm{FPR}}=108 \mu \mathrm{m}$, the diffraction order $\mathrm{m}=36$ for the low resolution AWG and 130 for the high resolution AWG. Figure 1 shows the microscope image of the low resolution AWG. The footprint of the low resolution and high resolution AWG is $0.45 \mathrm{~mm}^{2}$ and $1.5 \mathrm{~mm}^{2}$, respectively. This small device size can enable a SWIR miniature spectroscopy system based on the silicon photonics platform.
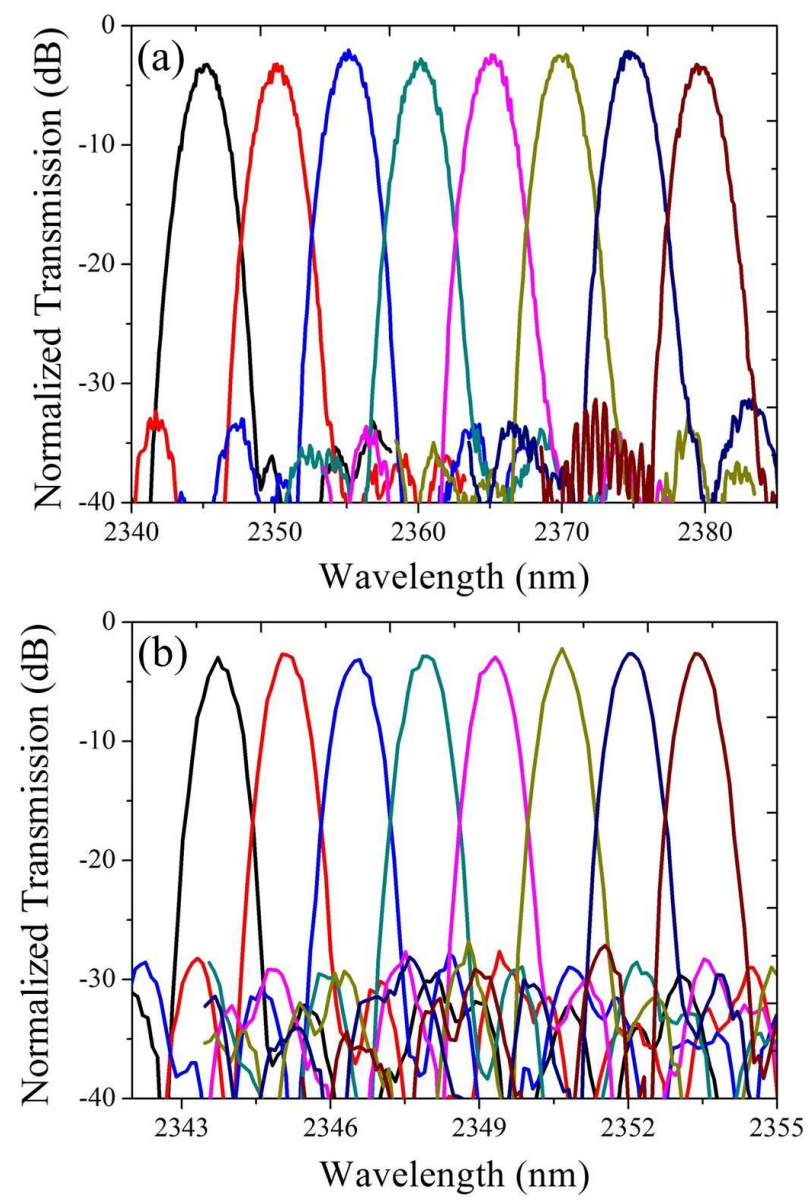

Figure 2. Transmission spectrum of the low resolution (a) and high resolution (b) AWG.

The AWG performance including insertion loss and crosstalk is characterized by measuring the transmission spectrum of every channel. TE-polarized light from a tunable SWIR laser (IPG Cr:ZnSe solid state laser) is coupled to a standard single mode fiber and then split by a $99 / 1 \%$ splitter. $1 \%$ of the input power is coupled to a photodetector, which can be used as a power reference. $99 \%$ of the input power is coupled to the AWG through a grating coupler and then collected by an optical spectrum analyzer (Yokogawa AQ6375). The measured transmission spectra of AWGs are normalized to a reference waveguide, and are shown in Figure 2 (a) and (b). From this figure, it is can be seen that the average insertion loss is around $2.5 \mathrm{~dB}$ and the crosstalk level is $-30 \mathrm{~dB}$ for the low resolution AWG. The high resolution AWG has an average insertion loss of about $2.8 \mathrm{~dB}$ and crosstalk level of $-25 \mathrm{~dB}$. The insertion loss and crosstalk of these AWGs are much lower than previously demonstrated $2 \mu \mathrm{m}$ wavelength AWGs based on strip waveguides. The performance is comparable with stateof-the-art SOI AWGs operating at telecommunication wavelengths. 


\section{HETEROGENEOUS TYPE-II QUANTUM WELL PHOTODETECTORS}

The heterogeneously integrated photodetectors consist of InP-based type-II quantum wells bonded to an SOI waveguide circuit as shown in figure 3 (a). In order to efficiently couple the light from the silicon waveguide to III-V active region, both the III-V waveguide and silicon waveguide are tapered. Figure 3(b) shows the schematic of the top view of the heterogeneously integrated devices. The length of the III-V waveguide is $150 \mu \mathrm{m}$, tapered from $1 \mu \mathrm{m}$ to $3.5 \mu \mathrm{m}$. The silicon waveguide underneath is a $400 \mathrm{~nm}$ thick rib waveguide etched $180 \mathrm{~nm}$ deep, tapered from $6 \mu \mathrm{m}$ to $3 \mu \mathrm{m}$. The light is confined in the silicon waveguide in the beginning of the double taper section, and then gradually couplse up to III-V waveguide when the photodetector mesa goes wider. Detailed information about the design of the photodetector can be found in [12].

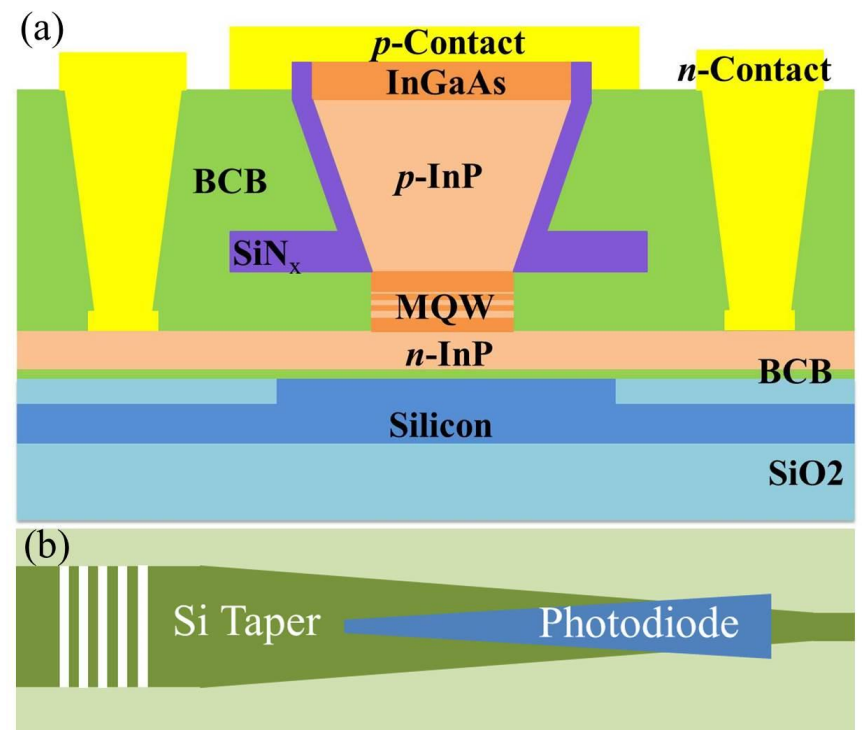

Figure 3. Schematic of the cross-section (a) and top view (b) of the InP-based type-II quantum well photodetector integrated on an SOI waveguide circuit.

The III-V epitaxial structure of the photodetector consists of a p-InGaAs contact layer, a p-InP cladding layer, an absorbing active region sandwiched between two separate confinement heterostructure ( $\mathrm{SCH}$ ) layers, and an $\mathrm{n}-\mathrm{InP}$ contact layer. The active region consists of six periods of a "W"-shaped quantum well structure, each separated by $9 \mathrm{~nm}$ tensile strained GaAsSb. Each quantum well consists of one $2.9 \mathrm{~nm} \mathrm{GaAsSb}$ layer confining the holes, surrounded by two $2.6 \mathrm{~nm}$ electron confining InGaAs layers. Specific information on the active region design can be found in Ref. 11, as it has been used for the realization of $2.4 \mu \mathrm{m}$ wavelength lasers. This III-V structure is grown on an InP substrate by a molecular beam epitaxy (MBE) system and then transferred to the patterned silicon wafer by adhesive bonding with a $100 \mathrm{~nm}$ thick BCB layer. Afterwards the InP substrate is removed by a $\mathrm{HCl}$ solution using InGaAs as etch stop layer.

Figure 4(a)-(h) shows the process flow of the heterogeneously integrated photodetector. After bonding and substrate removal, wet-etching of the p-InP and active region is used to process the photodetector mesas on the III-V membrane. The first process is to define a tapered waveguide with $1 \mu \mathrm{m}$ taper tip in a $\mathrm{SiN}_{\mathrm{x}}$ hard mask layer. Then the InGaAs contact layer is etched by ICP and the $p$-InP cladding layer is etched using a 1:1 $\mathrm{HCl}: \mathrm{H}_{2} \mathrm{O}$ solution. This anisotropic $\mathrm{HCl}$ wet etching of InP can generate undercut structures when the mesa is oriented along the [01-1] direction, which reduces the lithographic requirements of the tapered waveguides. For the lithography pattern with 1 um taper tip on the hard mask, the bottom width of the $\mathrm{p}$-InP after $\mathrm{HCl}$ etching is around $200 \mathrm{~nm}$ wide. After $p$-InP etching, subsequent wet-etching of the quantum well layers to the $\mathrm{n}$-InP layer is performed by a $\mathrm{H}_{3} \mathrm{PO}_{4}$ : Citric Acid: $\mathrm{H}_{2} \mathrm{O}_{2}: \mathrm{H}_{2} \mathrm{O}$ 1:1:20:70 solution. Afterwards, $\mathrm{Ni} / \mathrm{Ge} / \mathrm{Au}$ is deposited as n-contact. Then the $\mathrm{n}-\mathrm{InP}$ layer is etched by diluted $\mathrm{HCl}$ for device isolation. After metallization, 
DVS-BCB solution is spin coated on the sample to passivate the devices. After curing at $250^{\circ} \mathrm{C}$ for $1.5 \mathrm{~h}$, the $\mathrm{BCB}$ layer is etched by reactive ion etching (RIE) for the opening of the p-contact and n-contact. In the end, Ti/Au is deposited on the $\mathrm{n}$-contact and p-contact as probe pad. Fig. 4(i) shows a scanning electron microscope (SEM) image of the device cross section.

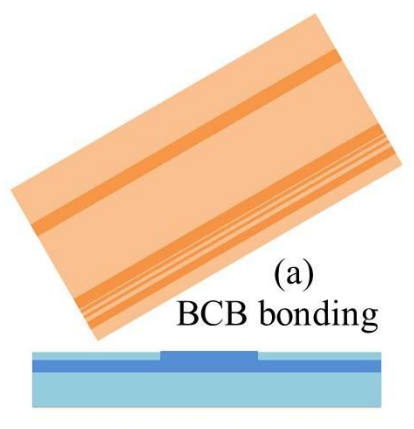

(d) Quantum well etching

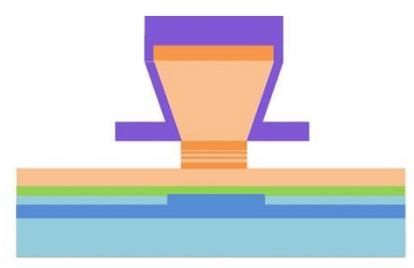

(g) BCB and $\mathrm{SiN}_{\mathrm{x}}$ etching

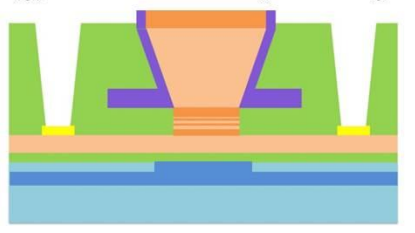

(b) Substrate removal

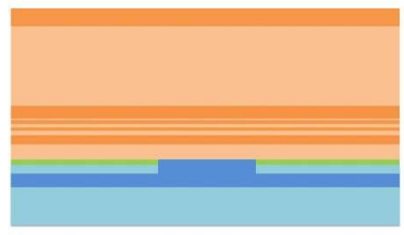

(e) N-contact metallization

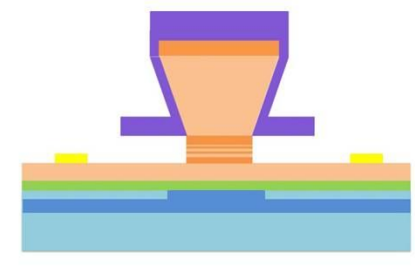

(h) Final contact metallization

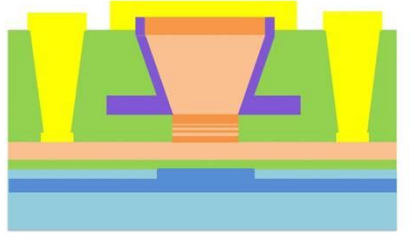

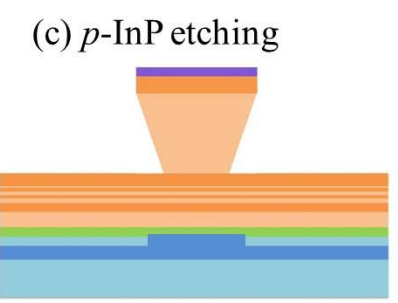

(f) BCB passivation

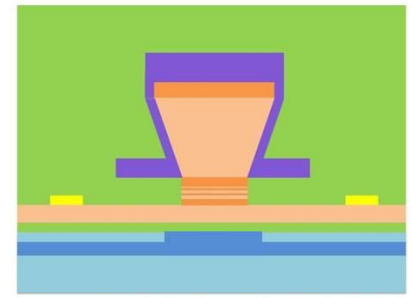

(i)

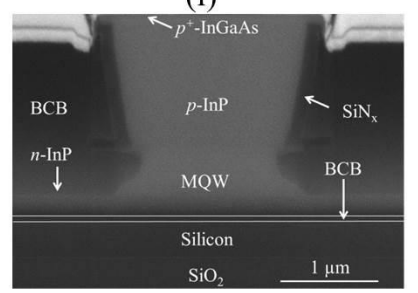

Figure 4. (a)-(h): Process flow for the hybrid InP-based type-II quantum photodetector, (i) SEM image of the cross section of the fabricated device.

The electrical characteristics of the heterogeneously integrated photodetectors were measured. Figure 5 shows the I-V curve at room temperature. The measured dark current under reverse bias of $0.5 \mathrm{~V}$ is $12 \mathrm{nA}$ (resulting in a current density of $0.17 \mathrm{~mA} / \mathrm{cm}^{2}$ ). As the reverse bias is increased, the dark current increases gradually but remains lower than $100 \mathrm{nA}$ at a reverse bias of $3.5 \mathrm{~V}$. This is 3 orders of magnitude lower than heterogeneously integrated GaSb-based photodetectors previously demonstrated by our group, enabling a higher sensitivity. This low dark current is attributed to the welldeveloped InP processes and timely BCB passivation of the photodetector mesa. Besides, passivation of the p-InP cladding layer using $\mathrm{SiN}_{\mathrm{x}}$ also can enable low dark current. 


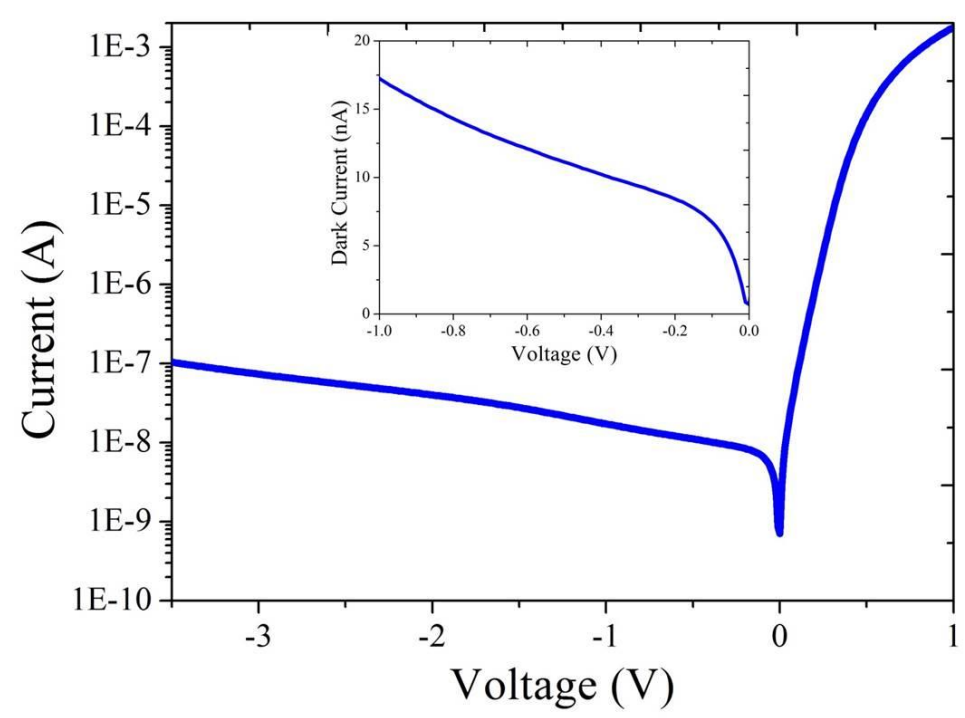

Figure 5. I-V curve of the heterogeneously integrated photodetector without light input. The inset picture shows the dark current of the heterogeneously integrated photodetector operating from $-1 \mathrm{~V}$ to $0 \mathrm{~V}$.

In the optical measurements, TE-polarized light is coupled to the silicon waveguide circuit by a $20 \mu \mathrm{m} \times 20 \mu \mathrm{m}$ grating coupler with a period of $1 \mu \mathrm{m}$. The measured peak coupling efficiency of the grating coupler is around $-9.5 \mathrm{~dB}$ at $2.35 \mu \mathrm{m}$ with $150 \mathrm{~nm} 3 \mathrm{~dB}$ bandwidth as shown in Figure 6(a). Figure 6(b) depicts the dependence of responsivity on reverse bias for a wavelength of $2.3 \mu \mathrm{m}$ and $2.4 \mu \mathrm{m}$. It can be found that the responsivity at $2.4 \mu \mathrm{m}$ is much more strongly dependent on reverse voltage than $2.3 \mu \mathrm{m}$. This can be attributed to strong electro-absorption at wavelengths close to the band gap wavelength of the active region. Figure 6(c) shows the optical responsivity as function of the input laser wavelength $(2.2$ $-2.42 \mu \mathrm{m}$ ) under reverse bias of $0 \mathrm{~V}$ and $0.5 \mathrm{~V}$. It can found that the heterogeneously integrated photodetector has a strong optical response at $0 \mathrm{~V}$ bias, which is attributed to a strong built-in field. From the grating coupler efficiency and fiberreferred responsivity measurement, the photodetectors have a waveguide referred responsivity higher than $0.5 \mathrm{~A} / \mathrm{W}$ over the 2.2-2.42 $\mu \mathrm{m}$ wavelength range. The measured peak internal efficiency is $1.2 \mathrm{~A} / \mathrm{W}$ at $2.32 \mu \mathrm{m}$ wavelength. 

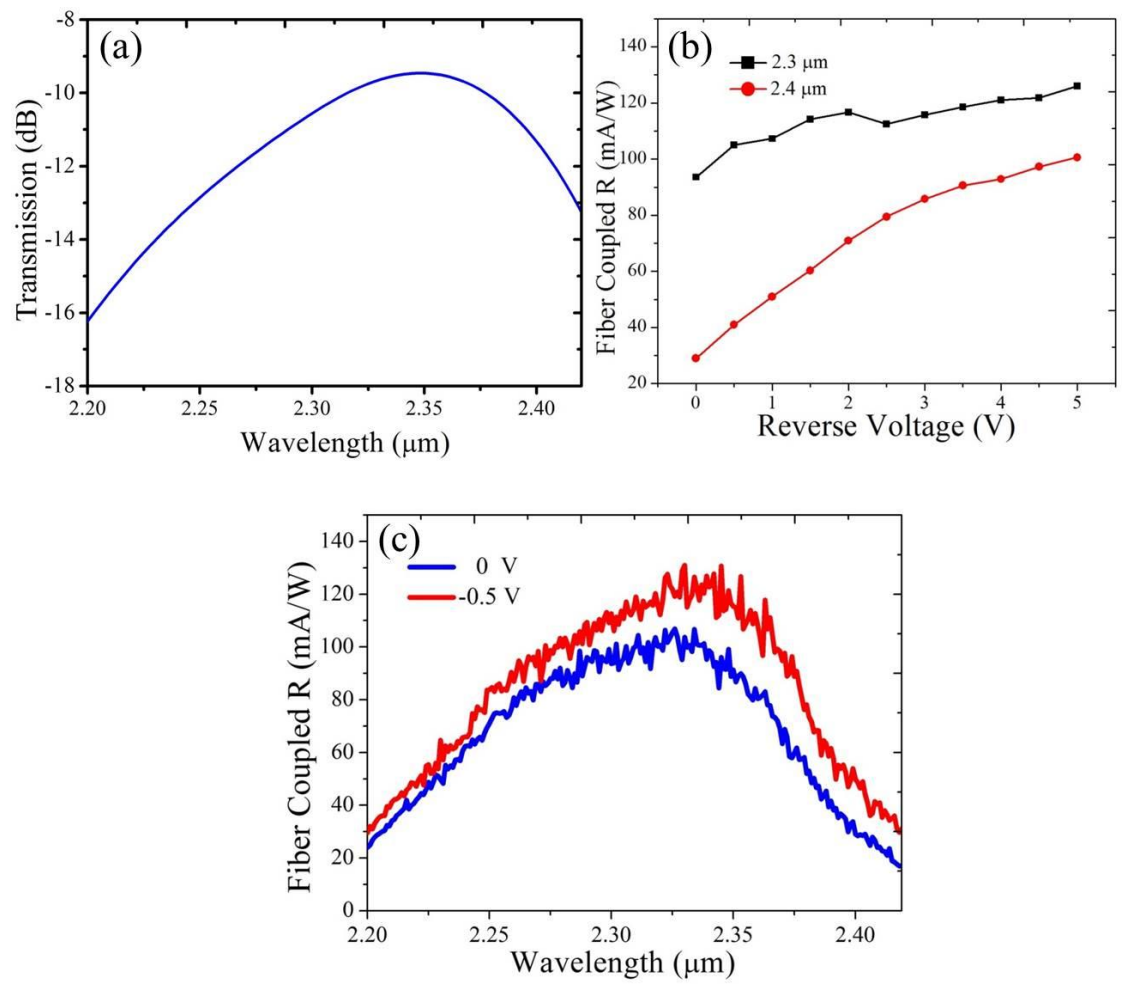

Figure 6. (a) The coupling efficiency of the grating coupler, (b) dependence of the fiber coupled responsivity on the reverse bias at wavelength of $2.3 \mu \mathrm{m}$ and $2.4 \mu \mathrm{m}$, (c) responsivity as function of the input laser wavelength under reverse bias of $0 \mathrm{~V}$ and $0.5 \mathrm{~V}$.

\section{CONCLUSION}

High performance SWIR AWGs and hybrid InGaAs/GaAsSb type-II quantum well photodetectors are fabricated and characterized. The low resolution $(5 \mathrm{~nm})$ and high resolution $(1.4 \mathrm{~nm})$ SWIR AWGs have a crosstalk of $-30 \mathrm{~dB}$ and -25 $\mathrm{dB}$, and insertion loss of $2.5 \mathrm{~dB}$ and $2.8 \mathrm{~dB}$, respectively. The photodetectors have a low dark current of $12 \mathrm{nA}$ and a high internal responsivity of $1.2 \mathrm{~A} / \mathrm{W}$ at a wavelength of $2.32 \mu \mathrm{m}$. The epitaxial stack for this photodetector integration can also be used to realize SWIR hybrid lasers, therefore enabling a fully integrated spectroscopic system.

\section{ACKNOWLEDGEMENT}

The author would like to thank S. Verstuyft for metallization processing help and L. Van Landschoot for SEM. This work was supported by the FP7-ERC project MIRACLE and the FP7-ERC-InSpectra.

\section{REFERENCES}

[1] L. S. Rothman, et al., "The HITRAN2012 molecular spectroscopic database," J. Quant. Spectrosc. Radiat. Transfer 130, 4-50 (2013).

[2] M. N. Petrovich, et al., "Demonstration of amplified data transmission at $2 \mu \mathrm{m}$ in a low-loss wide bandwidth hollow core photonic bandgap fiber," Opt. Express 21(23), 28559-28569 (2013). 
[3] G. Roelkens et al. , “ Silicon-based photonic integration beyond the telecommunication wavelength range, ” IEEE J. Sel. Topics Quantum Electron. 20(4), 394-404 (2014).

[4] G. Z. Mashanovich, et al., "Silicon photonic waveguides and devices for near- and mid-IR applications," IEEE J. Sel. Top. Quantum Electron. 21(4), 8200112 (2015).

[5] M. K. Smit and C. Van Dam, "Phasar-based wdm-devices: Principles, design and applications," IEEE J. Sel. Top. Quantum Electron. 2(2), 236-250 (1996).

[6] E. Ryckeboer, A. Gassenq, M. Muneeb, N. Hattasan, S. Pathak, L. Cerutti, J. B. Rodriguez, E. Tournié, W. Bogaerts, R. Baets, and G. Roelkens, "Silicon-on-insulator spectrometers with integrated GaInAsSb photodiodes for wide-band spectroscopy from 1510 to $2300 \mathrm{~nm}$,” Opt. Express 21(5), 6101-6108 (2013).

[7] G. Roelkens et al. , "II-V-on-Silicon Photonic Devices for Optical Communication and Sensing," Photonics 2(3), 9691004 (2015).

[8] Conley, B. et al. Temperature dependent spectral response and detectivity of GeSn photoconductors on silicon for short wave infrared detection. Opt. Express 22, 15639-15652 (2014).

[9] J. J. Ackert, D..J. Thomson, L. Shen, A. C. Peacock, P. E. Jessop, G. T. Reed, G. Z. Mashanovich, A. P. Knights., "High-speed detection above the telecommunication windows with monolithic silicon photodiodes," Nature Photon. 9, 393-396 (2015).

[10] G. Roelkens, L. Liu, D. Liang, R. Jones, A. W. Fang, B. R. Koch, and J. E. Bowers, "III-V/silicon photonics for onchip and intra-chip optical interconnects," Laser Photonics Rev. 4(6), 751-779 (2010).

[11] S. Sprengel, C. Grasse, P. Wiecha, A. Andrejew, T. Gruendl, G. Boehm, R. Meyer, and M. C. Amann, "InP-Based Type-II Quantum-Well Lasers and LEDs," IEEE J. Sel. Topics Quantum Electron. 19(4), 1900909-1900917 (2013).

[12] R.Wang, S. Sprengel, M.Muneeb, G. Boehm, R. Baets, M. C. Amann, and G. Roelkens, "2 $\mu \mathrm{m}$ wavelength range InP-based type-II quantum well photodiodes heterogeneously integrated on silicon photonic integrated circuits," Opt. Express" 23(20), 26834-26841 (2015). 